\title{
Visual contrast sensitivity in drug-induced Parkinsonism
}

\author{
C BULENS,* J D MEERWALDT, $\dagger$ G J VAN DER WILDT, $\ddagger$ C J KEEMINK $\ddagger$ \\ From the Departments of Neurology, Sint Franciscus Gasthuis, * University Hospital Dijkzigt, $\dagger$ and the \\ Department of Biological and Medical Physics, $\ddagger$ Erasmus University, Rotterdam, The Netherlands
}

SUMMARY The influence of stimulus orientation on contrast sensitivity function was studied in 10 patients with drug-induced Parkinsonism. Nine of the 10 patients had at least one eye with contrast sensitivity deficit for vertical and/or horizontal stimuli. Only generalised contrast sensitivity loss, observed in two eyes, was stimulus orientation independent. All spatial frequency-selective contrast deficits in 15 eyes were orientation dependent. The striking similarity between the pattern of contrast sensitivity loss in drug-induced Parkinsonism and that in idiopathic Parkinson's disease, suggests that generalised dopaminergic deficiency, from whatever cause, affects visual function in an analogous way.

We reported previously that a considerable proportion (25/39) of patients with Parkinson's Disease had visual Contrast Sensitivity (CS) loss.' The reversibility of CS losses under levodopa treatment suggested a dependence of visual function on dopaminergic transmission. ${ }^{2}$ Drug-induced Parkinsonism (DIP) is a generalised condition that resembles Parkinson's disease clinically and biochemically. If dopamine is a functional transmitter in the visual pathway, systemic administration of dopamine blockers will, in theory, affect CS function in a similar way as in Parkinson's disease. In the study reported here, we evaluated CS function in 10 patients with DIP.

\section{Methods and subjects}

Contrast sensitivity measurements The methods have been described elsewhere in detail. ${ }^{3}$ The stimuli were vertical and horizontal gratings with a sinusoidal profile.

Normal subjects: The normal group comprised 10 subjects, none of whom had any history of neurological or ophthalmological disorder. Subjects with known astigmatism were excluded. All had ophthalmological examination and were free of ocular disease. Snellen acuities, measured with refractive correction where necessary were $10 / 10$ or better. Patients: Ten previously untreated patients, who were alert, were selected. They were all receiving dopamine blockers

Address for reprint requests: C Bulens MD, Department of Neurology, Sint Franciscus Gasthuis, Kleiweg 500, 3045 PM Rotterdam, The Netherlands

Received 12 July 1988 and in revised form 16 October 1988. Accepted 21 October 1988
Table 1 Clinical summaries of 10 patients

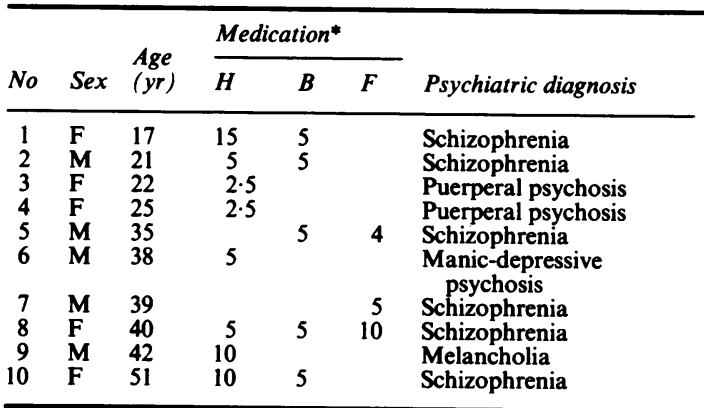

"Haloperidol (H) and benzhexol (B) in daily dose (mg); Fluspirilene (F) in weekly dose (mg).

(haloperidol and/or fluspirilene) for at least 3 weeks at the time of testing. All patients had drug-induced Parkinsonism, the severity of which was rated according to Hoehn and Yahr's stages categories. ${ }^{4}$ Clinical summaries and medication are listed in table 1 . To exclude possible effects of sedation on CS testing in the patient group, we also examined three agematched epileptic patients receiving phenytoin (dose $300 \mathrm{mg}$ / day). All patients had ophthalmological examination, including corrected visual acuity testing. Patients with known astigmatism were excluded. All patients and control subjects gave informed consent after the procedures and the aims of the study had been fully explained.

\section{Results}

Control group. The mean age of the control group was 35 years, (range, 23-51). The CS functions of the 20 control eyes were obtained for vertical and horizontal 


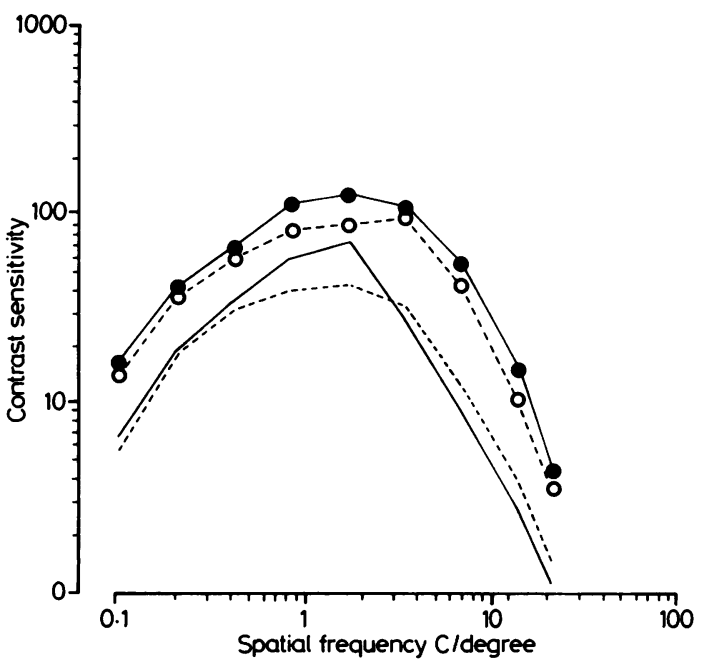

Fig 1 Mean contrast sensitivity curve of 20 normal eyes for vertical (filled circles) and horizontal gratings (open circles). Lines show $2 S D$.

gratings and the mean CS curves for both orientations are shown in fig 1 . Close inspection of individual CS curves in normal subjects reveals that at intermediate spatial frequencies a CS curve can diverge slightly from the pure inverted $U$ shape. Minor deflections were observed for vertical as well as horizontal stimuli. In 12 of the 20 measurements using vertical stimuli slight decrements were found, while in 10 of the 20 measurements using horizontal stimuli such decrements were observed. These decrements were expressed by their depths (decrease factor in $\log / \log$ ) and their widths (frequency ranges). The mean decrease factor (DF) of the 22 measurements was $1 \cdot 20$ (2 SD $=0 \cdot 22)$. These slight decrements in the individual CS function of the controls are completely within intrasubject variability.

Patient group. The mean age of the patient group was 33 years, (range, 17-51). All patients had Snellen acuities of $10 / 10$ or better. Rated according to Hoehn and Yahr they were all in stage II. The CS function for vertical and horizontal gratings of each of the patient's eyes was individually compared with the mean CS curves of the controls. A curve was judged abnormal when a CS deficit resulted in a displacement of (a part of) the curve below the control curve for more than two standard deviations. Intermediate frequency loss was taken for abnormal (notch defect) when the decrease factor (DF) was more than $1 \cdot 42$, being the mean DF in the normal group plus two standard deviations. The width of the notch was defined as the spatial frequency bandwidth over which contrast loss was observed (octaves). Two of the 10 DIP patients were retested 4 weeks later. The magnitude of
Table 2 Contrast sensitivity function for vertical and horizontal stimuli in 10 patients treated with dopaminergic blockers

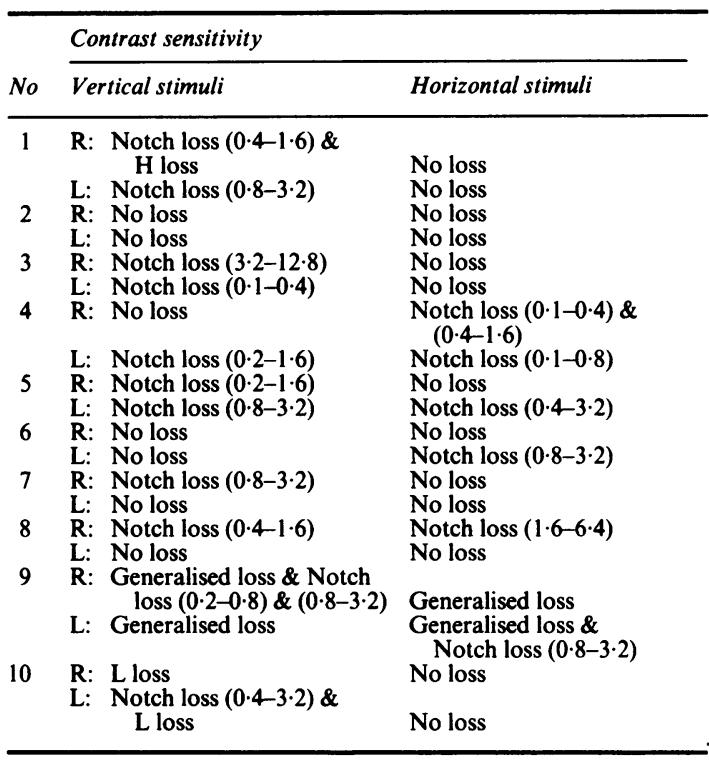

$\mathrm{R}=$ right eye; $\mathrm{L}=$ left eye. $\mathrm{H}$ loss $=$ high-frequency contrast sensitivity loss; $\mathrm{L}$ loss = low-frequency contrast sensitivity loss; Notch loss $=$ Notch contrast sensitivity loss (Spatial frequency cycles pero degree).

variability was $1 \cdot 46(2 \mathrm{SD}=0 \cdot 29)$. This test and retest consistency included the site and depth of the notch defects in these patients. CS function was obtained in 20 eyes. Only one patient had normal CS functions in both eyes for vertical and horizontal gratings. All the other nine patients had at least one eye with CS deficit for vertical and/or horizontal stimuli. Six of these nine patients had some CS losses in both eyes. The different types of CS loss for all patients are shown in table 2. CS losses affecting all the spatial frequencies tested were observed in both eyes of patient no 9. These deficits are referred to as generalised losses. All other CS losses were spatial frequency selective. The most frequent type of deficit, observed in 14 eyes, was a notch loss. In two eyes multifocal notch losses were found and in four other eyes they occurred in combination with other types of loss. Seventeen of the total number of 19 notches for either stimulus orientation occurred in the middle and low spatial frequency range (below 3.2 cycles/degree). Other types of spatial frequency selective CS loss were seen in three eyes. The loss affected selectively the high spatial frequency range in one eye, and the low spatial frequency range in two eyes. Although there was some overlap concerning orientation specificity in two eyes (nos 4,5 ), all spatial frequency-selective CS deficits were orientation dependent. The generalised losses in both eyes of 
Visual contrast sensitivity in drug-induced Parkinsonism
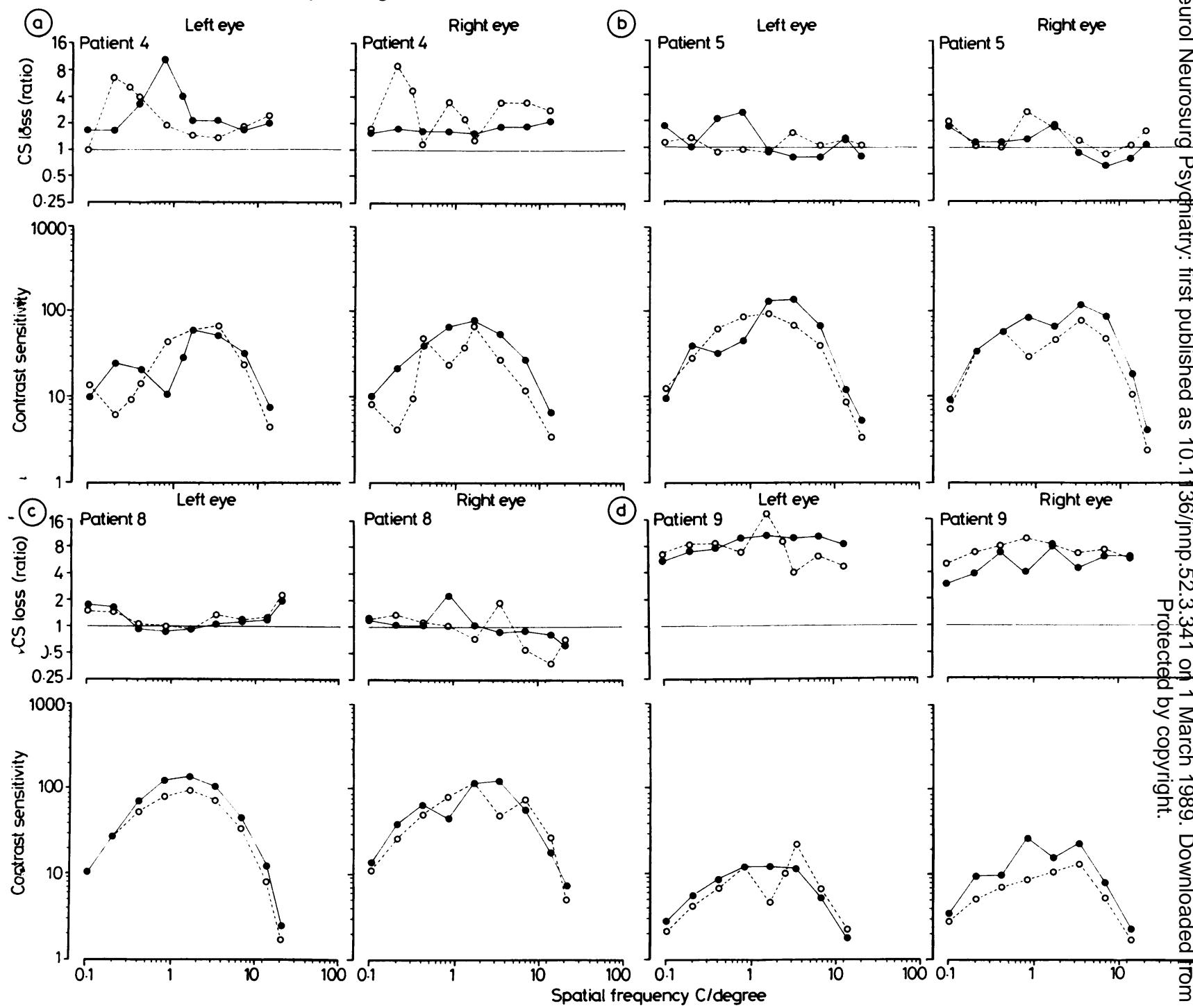

Fig 2 Examples of contrast sensitivity curves in four patients with drug-induced Parkinsonism. Key to symbols as in fig 1. The upper part of each figure shows the ratio of the altered contrast sensitivity to normal contrast sensitivity function for corresponding spatial frequencies. $(A)$ Patient 4. Left eye shows orientation-specific notch losses: $a 3$ octaves wide notch loss for horizontal gratings centred at $0 \cdot 2$ cycles/degree and a 3 octaves wide notch loss for vertical gratings at $0 \cdot 8$ cycles/degree. Right eye shows a notch loss centred at 0.2 cycles/degree and another notch loss centred at 0.8 cycles/degree for horizontal

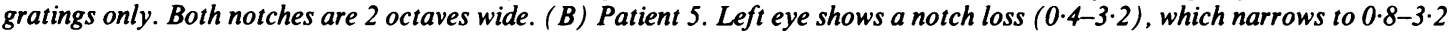
when CS is measured with vertical gratings. Right eye shows orientation-specific, 3 octaves wide notch loss centred at $0 \cdot 6$ cycles/degree for vertical gratings. (C) Patient 8. Left eye shows normal curves for both orientations. Right eye shows a 2 octaves wide notch loss centred at 0.8 and another of the same width at 3.2 cycles/degree, both orientation-specific, for vertical and horizontal gratings respectively. (D) Patient 9. Both eyes show orientation independent generalised loss. Left eye shows also a 2 octaves wide orientation-selective notch loss centred at $1.6 \mathrm{cycles} /$ degree. Right eye shows beside generalised loss a notch loss centred at 0.4 and another notch loss centred at $3 \cdot 2$ cycles/degree, both 2 octaves wide and orientation-selective. 
patient no 9 were orientation independent. Some examples of orientation-specific CS losses are shown in fig 2. The three epileptic patients all receiving phenytoin $(300 \mathrm{mg} /$ day) had normal curves for vertical as well as horizontal stimuli.

\section{Discussion}

Sine-wave grating CS determinations for vertical and horizontal orientations revealed CS loss in nine of the 10 patients with DIP. The most frequent type of deficit, observed in 14 eyes was a notch loss, which preferentially affected the middle and low spatial frequencies. All spatial frequency-selective CS loss was orientation dependent. Generalised loss, found in two eyes was orientation independent.

We reported previously that abnormal CS function occurred in a high frequency in Parkinson's disease.' In a subsequent paper ${ }^{3}$ we demonstrated that these losses were very stimulus orientation dependent. The most frequent type of CS loss was also a notch defect, which especially affected the middle spatial frequencies. Because orientation-sensitive neurons are not found peripheral to the primary visual cortex in primates, these orientation-dependent CS losses suggested cortical involvement in Parkinson's disease. In a recent study by Regan and Maxner, ${ }^{5}$ using temporally modulated stimuli at one spatial frequency ( 2 cycles/degree), orientation-selective loss was also demonstrated in six of 10 Parkinson's disease patients. CS loss was most marked at a temporal frequency of 4 to $8 \mathrm{~Hz}$. The maximum of CS loss was found for horizontally orientated stimuli. They proposed a possible explanation of their findings based on animal studies. In the visual cortical area V2 in the baboon there is a marked bias for neurons to prefer vertically orientated stimuli, while in area V1 there is a roughly similar preference for horizontal and vertical orientations. $^{6}$ Patients with a selective loss of CS for horizontal stimuli might have selective damage to the human equivalent of V1 striate cortex. Although comparison of our data with their findings is rendered difficult because of different techniques of CS measurement, the selective loss of CS for horizontal stimuli was not encountered in the present study and our previous report. ${ }^{3}$ The roughly equal CS losses for vertically and horizontally orientated stimuli, on the contrary, might indicate involvement of other visual cortical areas than the human equivalent of V1 striate cortex. Spatial and temporal "channels of vision" can apparently be affected differentially in a variety of ways in Parkinson's disease.

Monocular CS loss and important interocular differences of CS loss, however, could imply prechiasmal, possibly retinal involvement. Dopamine-sensitive cells are known to exist in the human retina, ${ }^{7}$ and it has been suggested that the dopaminergic deficiency associated with Parkinson's disease might result in peripheral visual dysfunction. ${ }^{8}$ Whether or not peripheral effects contribute to orientation-dependent CS loss in Parkinson's disease and DIP remains to be established.

A major assumption implicit in diagnosing CS deficits is the intra-subject test-retest consistency. We measured this by retesting CS in three DIP patients, 4 weeks later. The mean magnitude of variability was only $1.46(2 \mathrm{SD}=0.29)$, which means a great testretest consistency. The site and depth of the notch defects were highly reproducible. To minimise any possible effect of sedation on CS testing in our DIP patient group we also examined three epileptic patients receiving $300 \mathrm{mg}$ phenytoin per day. All three patients showed normal CS curves for vertical and horizontal grating stimuli.

Patients treated with neuroleptics like butyrophenones (haloperidol) and diphenylbutylpiperidines (fluspirilene) may develop a clinical picture which mimics Pakinson's disease very closely. DIP and Parkinson's disease are two conditions that share pharmacological, ${ }^{9}$ and biochemical ${ }^{10}$ characteristics. The fact that DIP is only produced by drugs that interfere with pre-synaptic storage of dopamine, or block post-synaptic dopamine receptors, indicates that dopamine deficiency is the biochemical basis of this clinical condition. Parkinson's disease and DIP are classically associated with motor manifestations, but the disorders are probably more generalised." Abnormalities of visual evoked potentials ${ }^{812} 13$ and CS function in Parkinson's disease indicate that the visual system is also involved.

There is a striking resemblance of the pattern of CS loss in drug-induced Parkinsonism to that in Parkinson's disease. This curious parallel suggests that generalised dopaminergic deficiency, from whatever cause, has similar effects on visual function. Our present results support the view that dopamine is a functional transmitter in the visual pathway.

The authors thank Professor H van Crevel, Department of Neurology, Academisch Medisch Centrum, Amsterdam, for his critical and stimulating comments.

\section{References}

1 Bulens C, Meerwaldt JD, van der Wildt GJ, Keemink CJ. Contrast sensitivity in Parkinson's disease. Neurology 1986;36:1121-5.

2 Bulens C, Meerwaldt JD, van der Wildt GJ, Van Deursen JBP. Effect of Levo-dopa Treatment on Contrast Sensitivity in Parkinson's Disease. Ann Neurol 1987;22:365-9.

3 Bulens C, Meerwaldt JD, van der Wildt GJ. Effect of stimulus orientation on contrast sensitivity in Parkin- 
son's disease. Neurology 1988;38:76-81.

4 Hoehn MH, Yahr MD. Parkinsonism: onset, progression, and mortality. Neurology 1967;17:427-42.

5 Regan D, Maxner C. Orientation-selective visual loss in patients with Parkinson's disease. Brain 1987;110: 415-32.

6 Kennedy H, Martin KAC, Orban GA, Whitteridge D. Receptive field properties of neurones in visual area 1 and visual area 2 in the baboon. Neuroscience 1985;14:405-15.

7 Frederick JM, Rayborn ME, Laties AM, Lam DMK, Hallyfield JG. Dopaminergic neurons in the human retina. J Comp Neurol 1982;210:65-79.

8 Bodis-Wollner I, Yahr MD, Mylin L, Thornton JT. Dopaminergic deficiency and delayed visual evoked potentials in humans. Ann Neurol 1982;11:478-83.

9 Ambani LM, van Woert MH, Bowers MB. Physostig- mine effects on phenothiazine-induced extrapyramidal reactions. Arch Neurol 1973;29:444-6.

10 Chase TN, Schnur JA, Gordon EK. Cerebrospinal fluid monoamine catabolites in drug-induced extrapyramidal disorders. Neuropharmacology 1970; 9:265-8.

11 Barbeau A, Campanella G, Butterworth RF, Yamada K. Uptake and efflux of ${ }^{14} \mathrm{C}$-dopamine in platelets: evidence for a generalised defect in Parkinson's disease. Neurology 1975;25:1-9.

12 Bodis-Wollner I, Yahr MD. Measurements of visual evoked potentials in Parkinson's disease. Brain 1978;101:661-71.

13 Gawel MJ, Das P, Vincent S, Clifford Rose F. Visual and auditory evoked responses in patients with Parkinson's disease. J Neurol Neurosurg Psychiatry 1981;44: 227-32. 